\title{
Un exemple des apports scientifiques des technologies de dynamique
} sédimentaire

\author{
A. GROVEL \\ Professeur d'Université, L.M.G., Université de Nantes
}

\section{Résumé}

Pour étudier une zone côtière aux fins d'aménagement, un éventail important de technologies a été mis en cuvre : sondage hydrographique, géophysique marine, cartographie par plongée, mesures de houles, de courants, de marées, corrélation houlemétéorologie, dragages et carottages, analyse physique et chimique des sédiments.

C'est l'utilisation d'un modèle mathématique de transport des sédiments, DIAMAX, qui a permis de faire une synthèse des divers résultats.

II en est sorti deux enseignements scientifiques : l'un concernant la géologie du quaternaire, l'autre concernant l'océanographie de la zone NORD-GASCOGNE.

\section{Situation}

La présente communication rapporte les études en nature dans les "coureaux de GROIX", zone située entre l'île de GROLX et la côte, à l'entrée du port de LORIENT, au Sud de la Bretagne (carte $\mathrm{n}^{\circ} 1$ ).

Il y a six milles nautiques entre l'île et la côte et cette île constitue une protection naturelle remarquable pour le chenal du port de LORIENT.

La profondeur d'eau moyenne est de 20 mètres et le marnage maximal est de 5,50 mètres.

Toute la côte de cette région est caractérisée par une alternance de pointes rocheuses granitiques et de longs cordons dunaires abritant des anses lagunaires.

Ces cordons disparaitraient sous l'action de la mer sans les travaux de protection de l'homme.

Le littoral recule en effet depuis 17000 ans, époque où le niveau de la mer était à 120 mètres au dessous du niveau actuel : c'est la transgression flandrienne postérieure aux dernières glaciations.

Au moment de la surrection de la chaîne des Pyrénées les mouvements de la plaque EUROPE ont fait travailler le massif armoricain, provoquant notamment dans la région qui nous intéresse un long plissement de $300 \mathrm{~km}$ de long, d'orientation WNW-ESE, très visible à terre sous le nom de "Synclinorium de Cornouailles".

De plus, le réchauffement du climat après la dernière glaciation quaternaire a provoqué des coulées de boue emballant de gros blocs de granite et les transportant sur de longues distances. Les pluies du climat océanique les ont ensuite dégagés ; ces blocs erratiques sont fréquents dans le Nord du massif armoricain, on n'en trouve jamais dans le Sud. Par contre, sur la côte de notre zone d'étude on trouve les surfaces planes caractéristiques du glissement de ces coulées post-glaciaires. 
Le sol sous-marin entre GROIX et la côte est caractérisé par la présence d'une vallée sous-marine, d'orientation WNW-ESE, de profondeur 35 à 40 mètres, ouverte sur le large : la fosse du TALUD.

Depuis 20 ans cette fosse est utilisée pour expédier vers le large les boues épurées de la station d'épuration de l'agglomération lorientaise, à l'aide d'une canalisation sousmarine.

D'autres études, concernant des projets d'aménagement non réalisés, ont été régulièrement poursuivies sur cette zone.

Or un travail récent de notre laboratoire (GHADRI, 1994) a confirmé la justesse des résultats scientifiques mis en lumière par les technologies de dynamique sédimentaire de ces diverses études, d'impact ou d'exécution.

On peut donc actuellement passer en revue les questions posées au sujet de cette fosse et les technologies utilisées pour-trouver les réponses.

\section{Questions}

- S'agit-il d'un accident tectonique (faille) ou d'un creusement ?

- Dans l'hypothèse d'un creusement, le phénomène est-il actuel ou fossile ?

- Joue-t-elle le rôle de guide d'onde pour certaines houles ?

- Canalise-t-elle certains courants ?

- Quel est son rôle dans le transport sédimentaire?

\section{Technologies utilisées}

a) Cartographie des fonds

Les premières cartes marines précises, de cette zone, datent de 1808 , exécutées par BEAUTEMPS-BEAUPRÉ lui même avec sondage au plomb et positionnement au cercle hydrographique. Ces cartes furent reprises en 1855, 1895 et 1939.

Les minutes au $1 / 5000^{\circ}$ sont intéressantes mais sont destinées à la navigation.

II a paru essentiel de relever à nouveau des cartes détaillées au sondeur ultrasonore $(45 \mathrm{kHz})$ et de les interpréter de façon géologique.

Le sondage détaillé de la fosse a révélé un bord Sud très escarpé et de direction WNW-ESE, c'est-à-dire parallèle à la ligne de plissement principale de la région.

Entre la fosse et la côte, près de la pointe du TALUD (voir fig. 2) on peut distinguer des effondrements, des micro-falaises de la taille du mètre qui font penser à un réseau de failles.

Enfin, aux alentours de la pointe du TALUD elle-même, la profondeur trouvée au sondeur est de 2 à 3 mètres inférieure à celle que les hydrographes d'autrefois trouvaient au plomb.

Des vérifications par plongée s'imposaient. 
b) Reconnaissances par plongée sous-marine

Des croquis du fond réalisés à la boussole de plongée et repérage par lâchers de bouées positionnées en surface au cercle hydrographique ont mis en évidence près de la pointe du TALUD (fig. $n^{\circ} 3$ ) :

- Des blocs arrondis de granite de 3 à 5 mètres de diamètre, analogue à ceux du Nord de la Bretagne (les plombs des anciens hydrographes devaient se glisser entre les blocs, alors que l'uitra-son se réfléchit sur les sommets).

- Une falaise d'un mètre de rejet, d'orientation WNW-ESE.

- Des ripple-marks dans le sable à 20 mètres de profondeur, correspondant à des houles de $\mathrm{SW}$.

Dans la passe de PORT-LOUIS (point $G$ sur la carte $n^{\circ} 1$ ), des blocs de même taille, tous aussi arrondis, avaient été décrits en plongée par $40 \mathrm{~m}$ de fond (GROVEL, 1970).

Dans la fosse elle-même des plongées ont permis de compléter les renseignements fournis par les dragages et les carottages et d'établir un croquis sédimentairé (fig. $n^{\circ} 4$ ).

c) Explaration géophysiaue

Un étinceleur de 48 joules spécialement étudié pour les petits fonds a permis de déceler entre les points $D$ et $G$ de la figure $n^{\circ} 1$ une vallée ancienne, comblée d'une dizaine de mètres de sédiments.

La coupe $A B$ peut être interprétée sur la figure $N^{\circ} 5$.

La sismique réflexion y détecte très bien tout un réseau de fractures et de failles qui ont joué après le dépôt des terrains 1,2 et 3 mais avant le dépôt des sédiments 4 , les apports flandriens. cependant.

La vallée fossile y est moins nette, masquée par la fracturation, mais apparaît

d) Ende de la houle

Elle comprend trois parties:

1/ Mesures in-situ :

Au point $\mathrm{H}$ (fig. $\mathrm{n}^{\circ} 1$ ), un houlographe à pression posé sur le fond a enregistré deux mois de houle de printemps qui ont servi d'étalonnage aux études statistiques.

\section{2/ Relation entre la houle en $H$ et la houle du large:}

Ce sont les plans de houle qui sont la fonction de transfert entre la houle du large et la houle au point $\mathrm{H}$ pour une direction et une période donnée.

d'énergie.

Ces plans de houle indiquent de plus où se situent les concentrations

Le rôle de la fosse est généralement celui d'un dioptre plan : pour aucune direction il n'y a renforcement de la houle au niveau de la fosse. 
L'action de creusement des fonds n'est donc pas dû à cette cause.

Le plan des houles du Sud Ouest de $T=11$ s (fig. n ${ }^{\circ} 6$ ), montre bien par contre le rôle de l'île de GROIX, et combien la zone entre GROIX et la côte, bien abritée, est du point de vue sédimentologique essentiellement régie par les courants.

\section{3/ Corrélation houle du large et météorologie}

Une méthode de prévision des houles, le modèle SMB, appliquée a postériori aux tempêtes de 10 années de bulletins météorologiques a permis de situer les houles annuelle, decennale, centenale au point $\mathrm{H}$. (voir figure $\mathrm{n}^{\circ}$ )

Sur le plan géologique c'est évidemment la houle dont la probabilité d'apparition est le siècle qui est la plus intéressante.

Au point $H$ c'est une houle de 10 mètres de hauteur, de période 10-11 secondes, dont la direction sera W-SW, direction qui coïncide avec celle des vents locaux les plus fréquents et les plus forts.

En dehors de l'abri de l'île de GROIX c'est la houle qui transportera les grains de sable les plus gros sur le fond.

e) Etude des courants

Les courants de surface étaient déjà bien connus grâce aux travaux du Service Hydrographique de la Marine.

Pour relier courants et sédiments, il fallait aller plus loin, notamment dans la connaissance de la répartition verticale des vecteurs courants.

Autour de la fosse du TALUD et au milieu de celle-ci trois mouillages de courantographe ont été réalisés, avec, en complément, quatre stations au courantomètre pour la structure verticale. été exploitées.

Le long de la côte, entre $D$ et $G$ (fig. 1), plus d'un an de mesures de courants ont

Par traitement statistique, la vitesse maximale du courant de marée a été reliée au coefficient de la marée. Une analyse harmonique a permis d'extraire les courants de marée des mesures des courantographes et, par différence, de faire apparaître les courants non périodiques.

D'habitude, par cette méthode, on ne trouve que les courants dits "de dérive", liés aux vents locaux, et superficiels.

Là, une autre population de courants a été détectée :

- Courants de masse, comme le courant de marée,

- Apparaissant lorsque les vents d'Ouest, dominants, faiblissent ou s'annulent,

- Apparaissant après une surcote de la marée et accompagnant la décroissance de hauteur jusqu'à la hauteur prédite,

- Dirigés vers l'Ouest.

Il s"agissait, bien sûr, de courants dits "de pente" dont la présence pouvait seule expliquer la complexité de certaines coupes verticales de flot et de jusant (voir fig. 8) 
Les enregistrements de courants au fond de la fosse vont de plus confirmer l'existence d'un courant résiduel vers l'Ouest. large.

Ce courant de pente se révélait donc un évacuateur de sédiments très fins vers le

Pour mieux cerner son importance, il fallait étudier la pente dynamique de la mer, c'est-à-dire les surcotes.

\section{f) Etude de la marée}

Le L.M.G. de Nantes a entrepris sur la période 1988-1994 une série de réflexions sur la "marée de l'ingénieur génie civil". Parmi ces travaux une thèse a mis en lumière le rôle des surcotes (GHADRI 1994).

Si l'on appelle "surcote" la différence de hauteur entre la marée mesurée (à pleine mer) et la marée prédite, la correction barométrique étant effectuée, on peut présenter les surcotes maximales, d'Est en Ouest :

à Saint-Nazaire (embouchure de la LOIRE), Port-Tudy (île de GROIX),

Le Conquet (extrême pointe du Finistère Nord)

\begin{tabular}{|c|c|c|c|}
\cline { 2 - 4 } \multicolumn{1}{c|}{} & Saint-Nazaire & Port-Tudy & Le Conquet \\
\hline 1976 & $110 \mathrm{~cm}$ & $64 \mathrm{~cm}$ & $59 \mathrm{~cm}$ \\
\hline 1978 & $117 \mathrm{~cm}$ & $76 \mathrm{~cm}$ & $72 \mathrm{~cm}$ \\
\hline 1979 & $90 \mathrm{~cm}$ & $44 \mathrm{~cm}$ & $60 \mathrm{~cm}$ \\
\hline 1980 & $/$ & $55 \mathrm{~cm}$ & $69 \mathrm{~cm}$ \\
\hline 1982 & $96 \mathrm{~cm}$ & $66 \mathrm{~cm}$ & $64 \mathrm{~cm}$ \\
\hline 1984 & $78 \mathrm{~cm}$ & $60 \mathrm{~cm}$ & $58 \mathrm{~cm}$ \\
\hline 1985 & $78 \mathrm{~cm}$ & $50 \mathrm{~cm}$ & $56 \mathrm{~cm}$ \\
\hline 1986 & $63 \mathrm{~cm}$ & $46 \mathrm{~cm}$ & $44 \mathrm{~cm}$ \\
\hline
\end{tabular}

La pente entre Saint-Nazaire et Port-Tudy est un phénomène constant par vent d'afflux : entre Le Conquet et Port-Tudy ce phénomène est plus variable.

Cette pente dynamique existe donc bel et bien dans les surcotes, et après disparition de la cause, le vent d'afflux de secteur Ouest, le courant de pente s'établit vers l'Ouest accompagnant le rétablissement du niveau habituel.

Entre Groix et la côte il s'agit donc d'un passage privilégié de ce courant de pente, notamment en fosse du TALUD. 


\section{g) Sédimentologie et sédimentologie dvnamique}

Quand on applique aux sables superficiels de la zone ouverte aux houles d'Ouest le programme DIAMAX de notre laboratoire (GROVEL, 1990), on s'aperçoit que la houle centenale ne peut remuer que $50 \%$ de la population des grains.

De même, de G en D (fig. 1), une étude serrée (GROVEL, THOMAS 1982) a montré que les courants de vidange de l'estuaire renvoyait vers le large les sables fins que la houle pouvait apporter, mais étaient impuissants vis à vis des sables grossiers sousjacents. De plus, en D, cette action s'atténue jusqu'à cesser.

Enfin, les carottages en fosse du TALUD montrent que les coquilles brisées qui tapissaient le fond en 1808 sont recouvertes actuellement par 20 à $50 \mathrm{~cm}$ de vase fine.

L'analyse chimique complète, le dosage de la matière organique, les rayons $\mathrm{X}$, le dosage du calcaire, comparés avec les mêmes résultats sur les vases de la rade de LORIENT confirment qu'il s'agit là de la partie la plus fine et la plus organique des vases de la rade.

\section{Interncétation}

La sédimentologie dynamique permet donc de répondre aux questions posées en reprenant les différents résultats sur cette zone.

La fosse du TALUD est bien une vallée fossile, la partie la plus ennoyée du paléo-lit du BLAVET, fleuve qui traverse la rade de LORIENT.

Cette vallée n'a pu être creusée par les houles ou courants actuels ; c'est un torrent à l'époque pré-flandrienne qui a profité des nombreuses failles pour orienter son cours vers l'Ouest.

De même, les sédiments sableux qui tapissent les fonds entre GROTX et la côte ne peuvent être mobilisés par les houles ni les courants actuels. Ce sont donc des reliques de conditions hydrauliques plus violentes.

Actuellement, seuls les sédiments fins sont transportés en suspension et un courant de pente canalisé par la fosse du TALUD, seul courant résiduel, les entraine vers l'Ouest, le large, c'est-à-dire la grande vasière.

\section{Conclusion}

Pour être interprétable une étude de sédimentologie dynamique doit mettre en cuvre de nombreuses technologies, mais seuie l'étude du transport sédimentaire permet de trancher entre différentes hypothèses de travail.

Ces travaux divers sur cette zone ont permis de dégager les résultats scientifiques suivants :

- les blocs erratiques, fréquents en Bretagne Nord, existent aussi en Bretagne Sud mais à une altitude inférieure puisqu'ils sont actuellement recouverts par la mer,

- il existe sur le littoral Sud de la Bretagne un courant de pente, se produisant après les surcotes et dont la présence est certaine entre l'embouchure de la Loire et l'île de Groix. 


\section{Références}

BONNEFILLE, PERNECKER (1966) : Le début d'entraînement des sédiments sous l'action de la houle. Bulletin Centre de Recherche et d'Essais, Chatou 15; pp.27-32.

GHADRI H. (1994) : La Marée de l'Ingénieur du Génie Civil. Prévision des surcotes. Thèse de doctorat. Université de Nantes.

GROVEL A. (1970) : Etude d'un estuaire dans son environnement. Thèse d'état. Université de Nantes.

GROVEL A., THOMAS P. (1982): Sols frottants mobiles et mobilisables sous l'action de la houle en géotechnique marine. Revue française de Géotechnique, 28 ; pp. 55-63.

GROVEL A. (1990) : L'utilisation en géotechnique marine de modèles mathématiques de transport, Compte-rendus $6^{\circ}$ Congrès International de l'AIGI, BALKEMA 5, pp. 36053607 . 


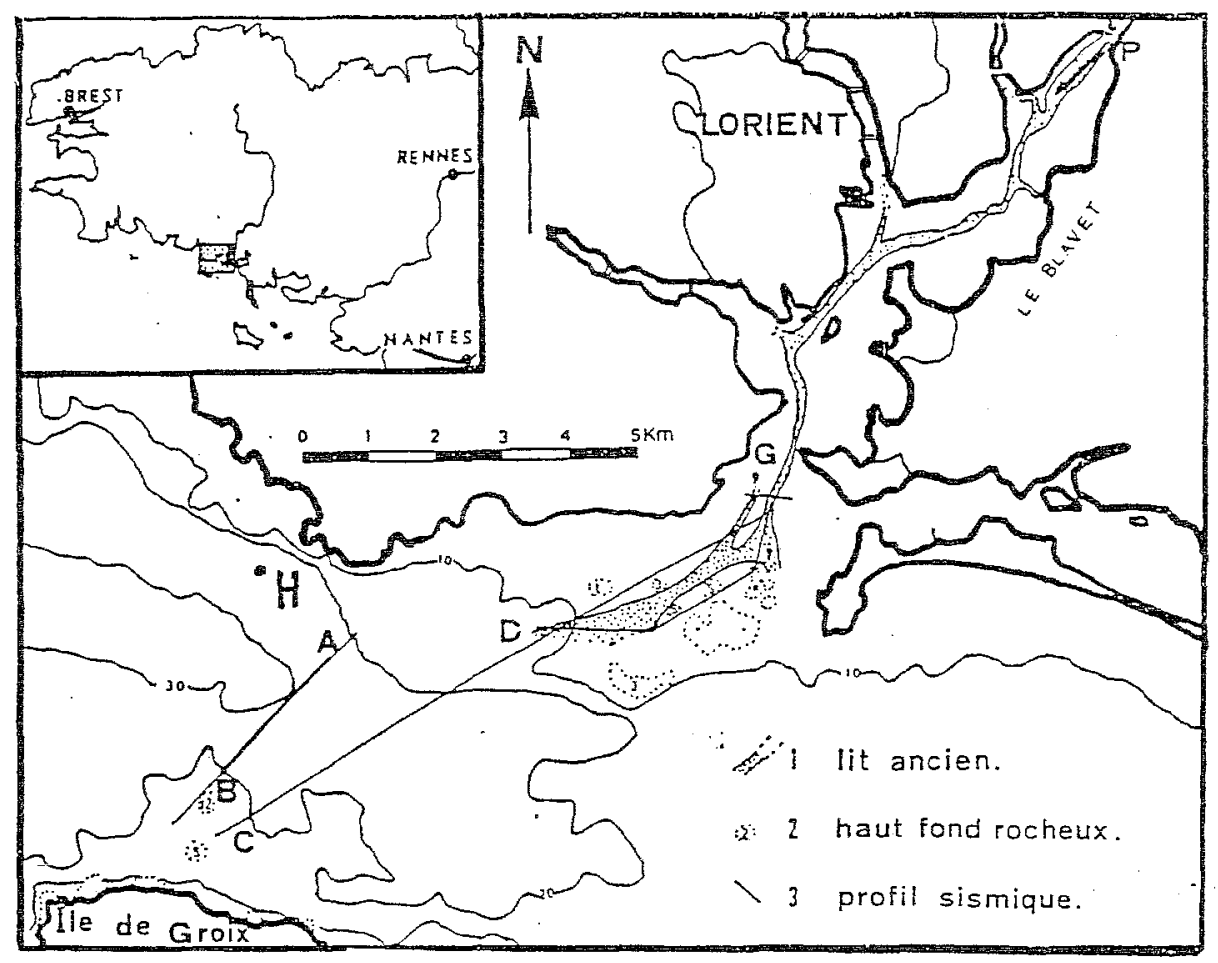

Fig. 1 : Situation générale.
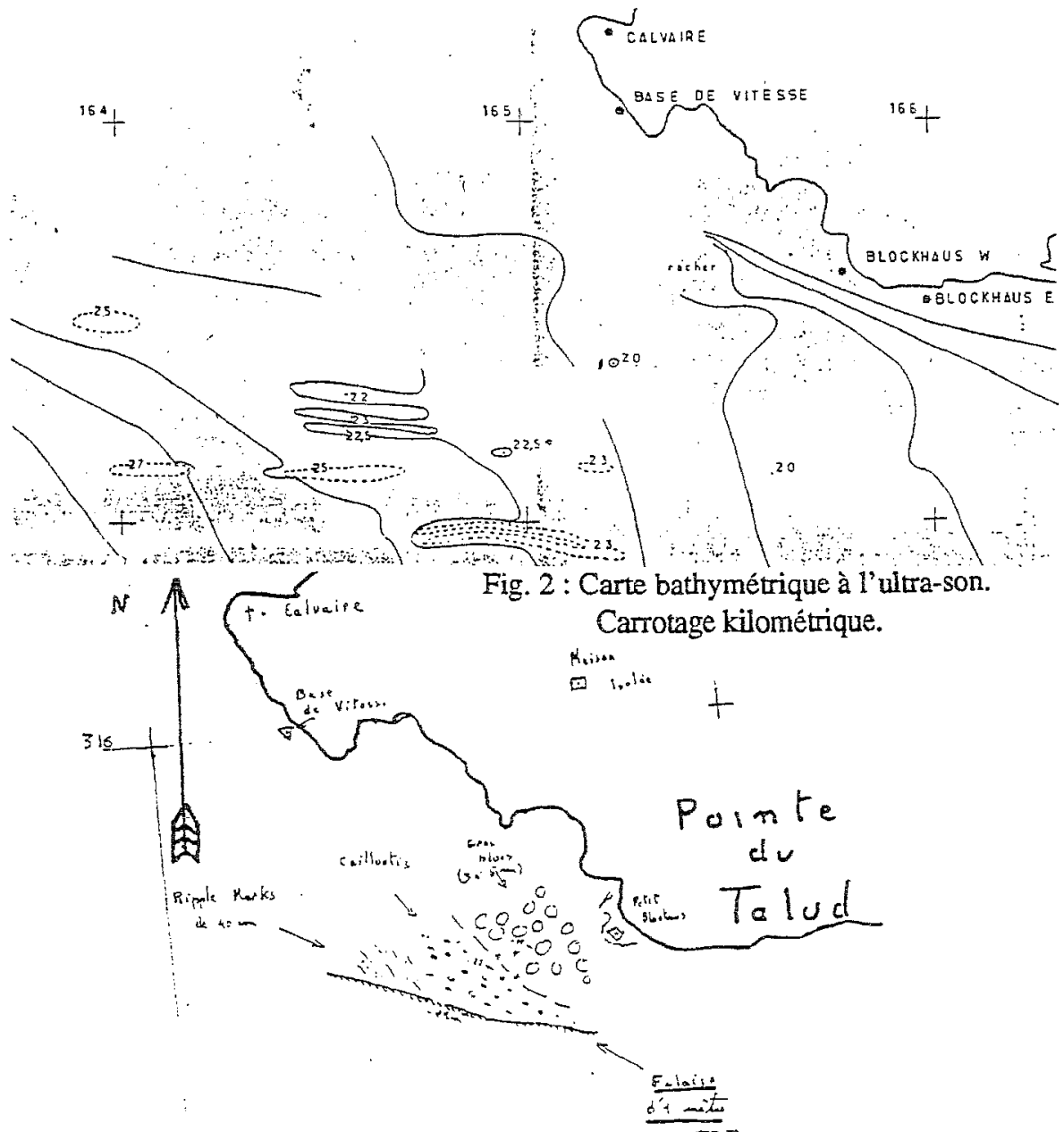

Fig. 3 : Croquis de plongée. (Y. MOIGN)

Carrotage kilométrique LAMBERT. 

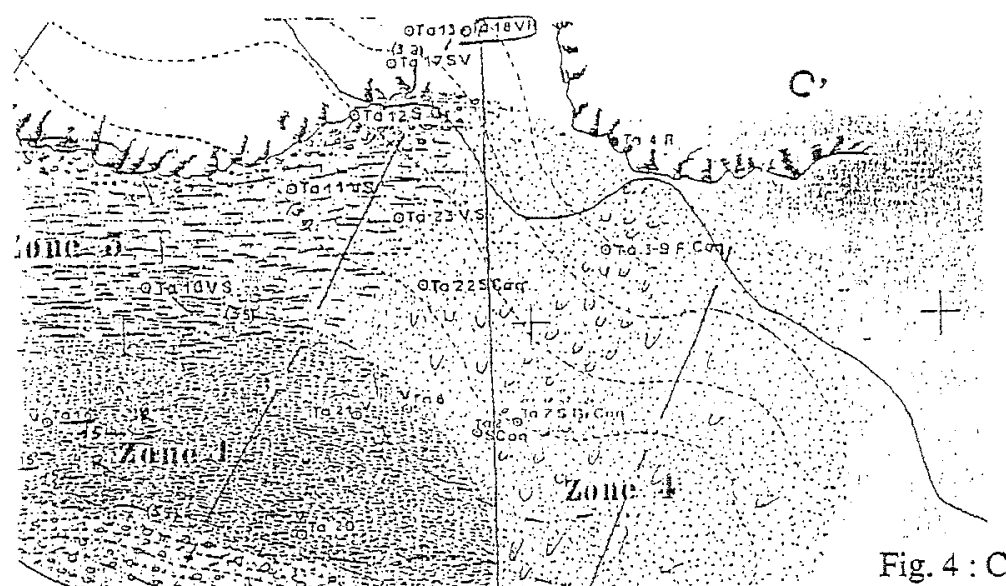

Fig. 4 : Croquis sédimentologique de la Fosse du TALUD.

Carrotage kilométrique LAMBERT.
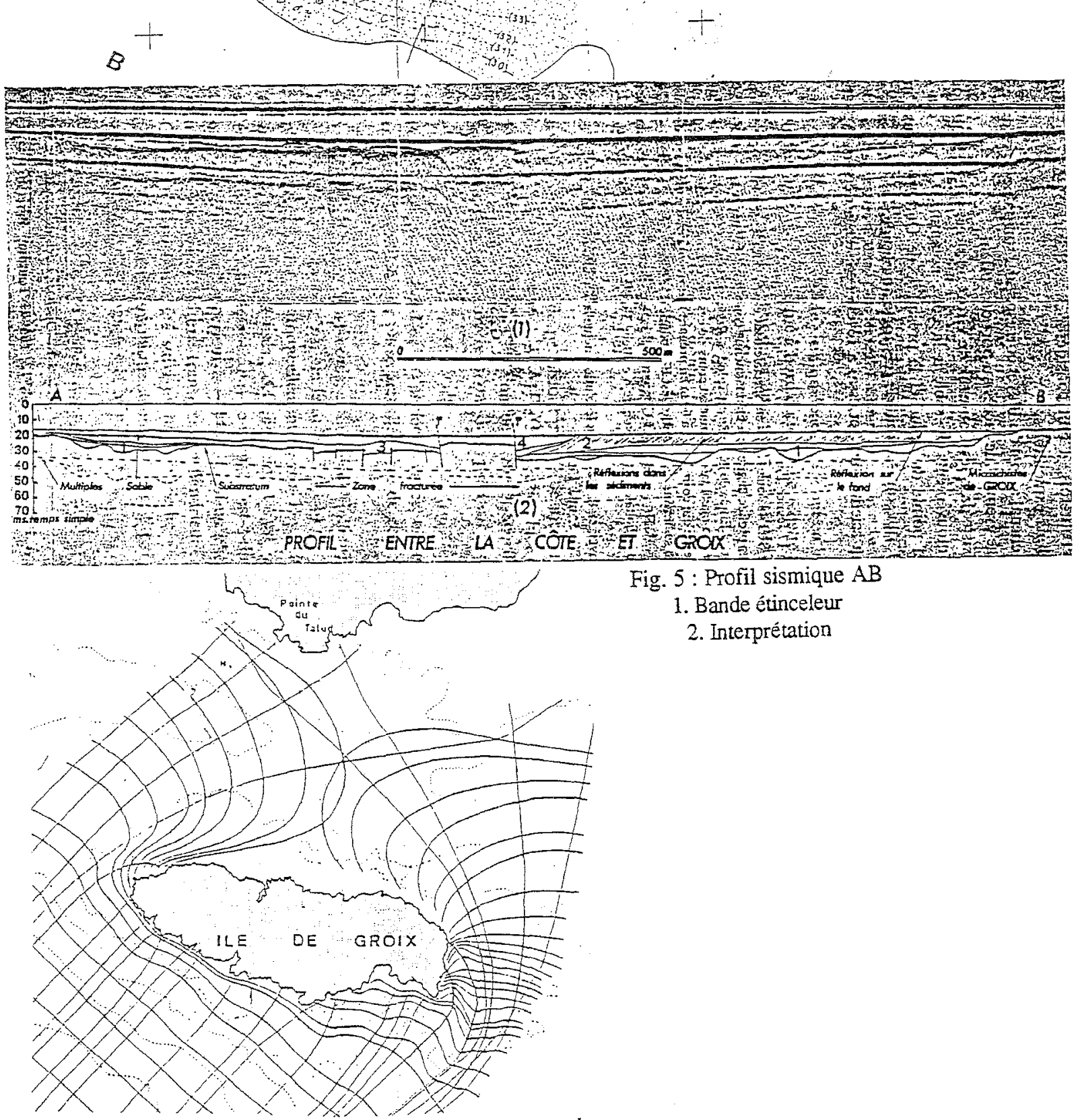

Fig. 5 : Profil sismique $A B$

1. Bande étinceleur

2. Interprétation

Fig. 6: Réfraction d'une houle SW de période 11 secondes. 


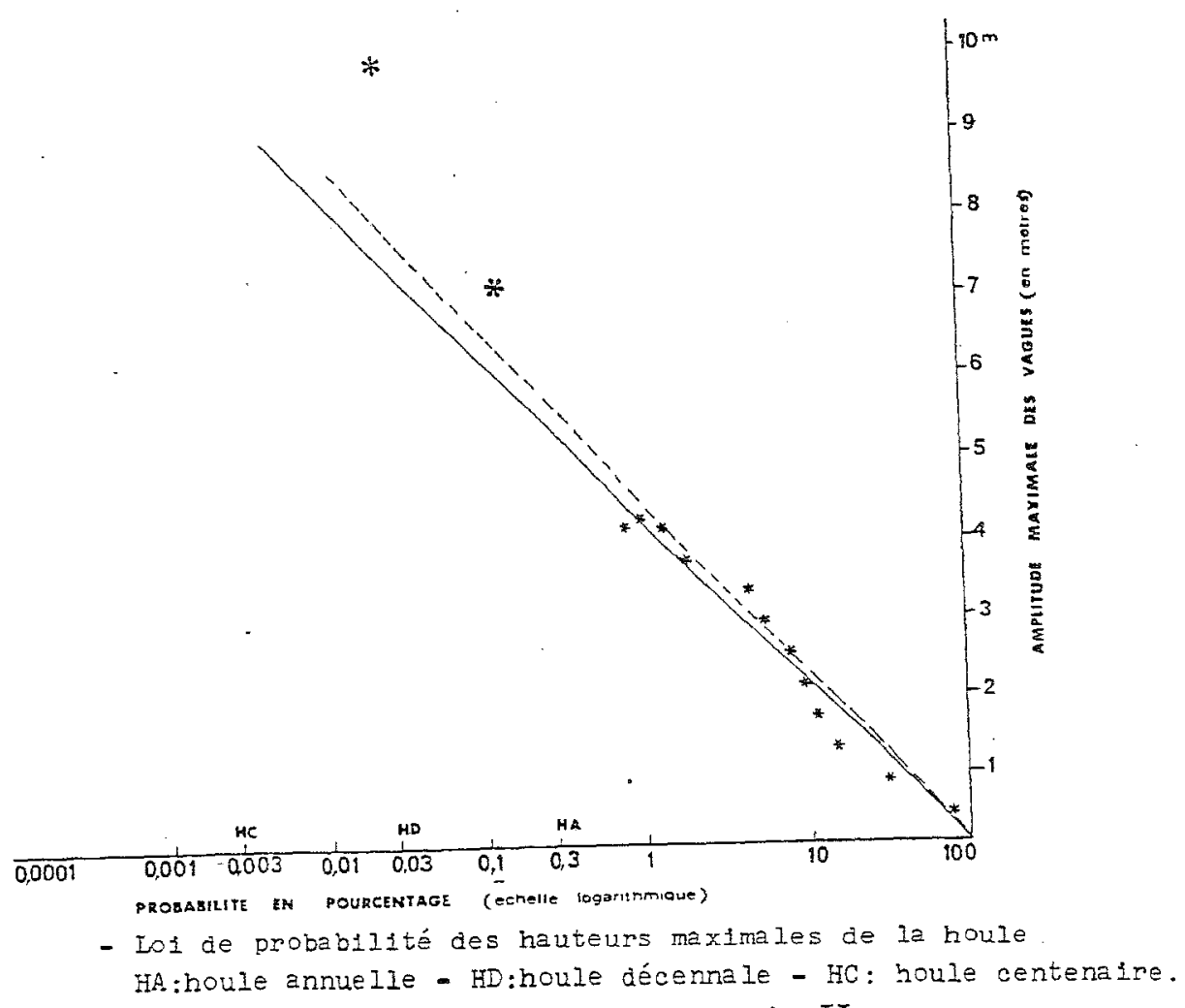

Fig. 7 : Prévision des houles au point $\mathrm{H}$.

Profils verticaux de vitesse.

Coef : 00 Mi-jusone

w

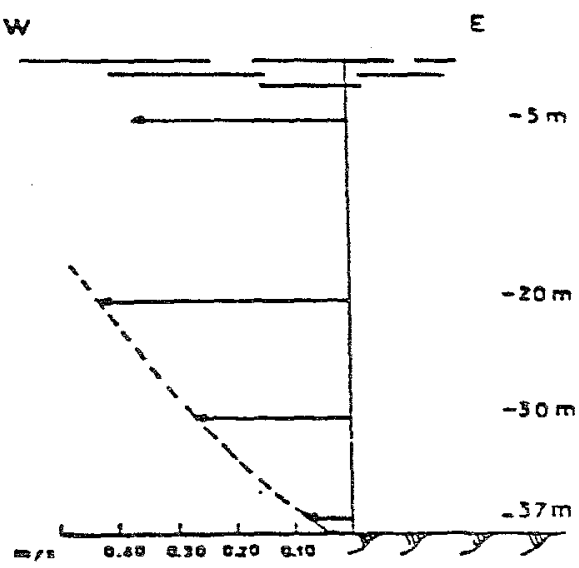

Coef: 76 -Fiol

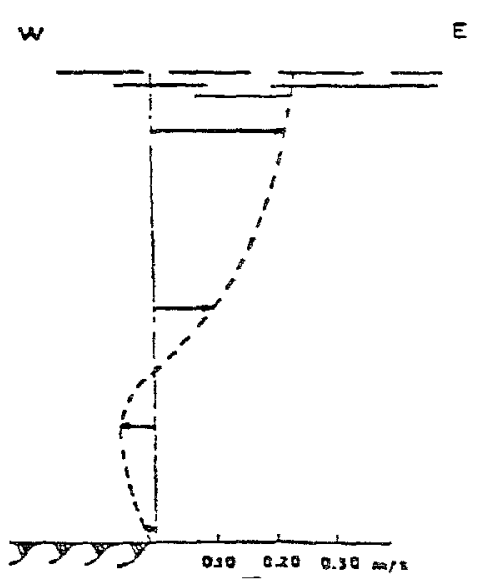

Fig. 8 : Exemple de la complexité des courants. 